\title{
Characterization of acoustic droplet formation in a microfluidic flow-focusing device
}

\author{
Yin Nee Cheung and Huihe Qiu* \\ Department of Mechanical Engineering, The Hong Kong University of Science and Technology, Clear Water Bay, Kowloon, Hong Kong
}

(Received 17 June 2011; revised manuscript received 24 September 2011; published 12 December 2011)

\begin{abstract}
Local control of droplet formation with acoustic actuation in a microfluidic flow-focusing device is investigated, and the effects of acoustic voltage, frequency, flow-rate ratio, fluid viscosity, and flow vorticity are characterized. Acoustic actuation is provided to affect droplet breakup in the squeezing regime by imposing periodic oscillation to the fluid-fluid interface and, therefore, a periodic change in its curvature at the cross-junction of the device. Time reduction is observed for the three key stages of droplet breakup in the squeezing regime: dispersed phase flow-front advancement into the orifice, pressure buildup upstream and within the orifice together with liquid inflation downstream, and finally the thinning and pinch-off of the liquid thread. It is found that acoustic actuation has less of an effect on droplet size for the continuous phase with a higher viscosity due to the restrained interfacial vibration under a high shear stress environment. Periodic velocity flow fields within the dispersed phase at different phases of one oscillation cycle are calculated based on the results from phaseaveraged microresolution-particle-image velocimetry ( $\mu$ PIV). The oscillation paths for the points of maximum vorticities of phase-averaged velocity components are traced, which reveals that the motion is mainly along the $y$ direction.
\end{abstract}

DOI: 10.1103/PhysRevE.84.066310

PACS number(s): 47.55.db, 47.55.df, 47.61.Jd, 43.25.+y

\section{INTRODUCTION}

Droplet and bubble formation processes based on flowfocusing geometry, T-shaped geometry, or hydrodynamic flowfocusing has drawn much attention in the past decade due to the high monodispersity on the droplets or the bubbles formed using such methods [1-12]. In these devices, droplet size was mainly controlled by the flow-rate ratio $\varphi$ between the two immiscible fluids:

$$
\varphi=\frac{Q_{d}}{Q_{c}}
$$

where $Q_{d}$ and $Q_{c}$ are the flow rates of the dispersed phase and the continuous phase, respectively.

Three common regimes reported for the droplet formation in a flow-focusing device include the squeezing or geometrycontrolled regime $[1,2,10,11]$, the dripping regime $[1,11]$, and the jetting regime $[1,11]$. These three regimes occur in different capillary numbers $\mathrm{Ca}$, in which the capillary number is usually defined based on the dynamic viscosity of the continuous phase $\mu_{c}$, the interfacial tension $\gamma$, and the average velocity of the continuous phase $u_{c}$ :

$$
\mathrm{Ca}=\frac{\mu_{c} u_{c}}{\gamma}=\frac{\mu_{c} Q_{c}}{\gamma L_{c} h},
$$

where $L_{c}$ and $h$ are the width and depth of the rectangular channel in which the continuous phase is flowing through, as shown in Fig. 1. Another definition, given by Anna et al. [11], takes the effective elongation rate $G=\Delta V / L_{c}$ into account, where $\Delta V=V_{r}-V_{c}$ is the velocity difference between the upstream velocity $V_{c}$ and the velocity at the orifice $V_{r}$, and $L_{c}$ is the length of the gap from the end of the inner channel to the start of the

\footnotetext{
*Author to whom all correspondence should be addressed: meqiu@ust.hk
}

orifice:

$$
\mathrm{Ca}^{\prime}=\frac{\mu_{c} G\left(L_{d} / 2\right)}{\gamma}=\frac{\mu_{c} L_{d} \Delta V}{2 \gamma L_{c}}=\frac{\mu_{c} L_{d} Q_{c}}{2 \gamma h L_{c}}\left[\frac{1}{L_{r}}-\frac{1}{2 L_{c}}\right],
$$

where $L_{d}$ is the width of the channel in which the dispersed phase is flowing through and $L_{r}$ is the width of the orifice.

Details of the breakup mechanism in the squeezing regime were discussed by Garstecki et al. [2,10]. This regime occurs at a low capillary number $\mathrm{Ca}$, in which the liquid thread of the dispersed phase first advances and fills up the orifice of the flow-focusing geometry, thus causing a pressure buildup upstream and within the orifice. The liquid thread is then "squeezed," and since this squeezing process is slow in comparison with the relaxation of the interfacial energy, the liquid thread then collapses in a series of equilibrium states. Finally, the liquid thread breaks rapidly when the unstable state is reached and the flow front of the dispersed phase retracts upstream of the orifice and the whole process starts again.

In the dripping regime, droplet breakup is caused by the Rayleigh capillary instability [13]. The flow front of the dispersed phase advances up and stays within the orifice. There is no retraction of the flow front after the breakup of each droplet. This phenomenon occurs at a capillary number higher than the squeezing regime, and the droplet sizes formed are also smaller.

Transition to the jetting regime occurs as the capillary number continues to increase. The droplet breakup mechanism is also caused by the Rayleigh capillary instability, but it is more difficult to control. In addition, the flow front of the dispersed phase extends further beyond the exit of the orifice and is maintained as a long liquid thread. The size of the droplet formed in this regime is larger than that in the dripping regime, but the size distribution is not monodisperse.

Several groups of researchers have attempted to provide local control together with the flow-rate ratio $\varphi$ upon extending the droplet size distribution [14-21]. Kim et al. [14] and 


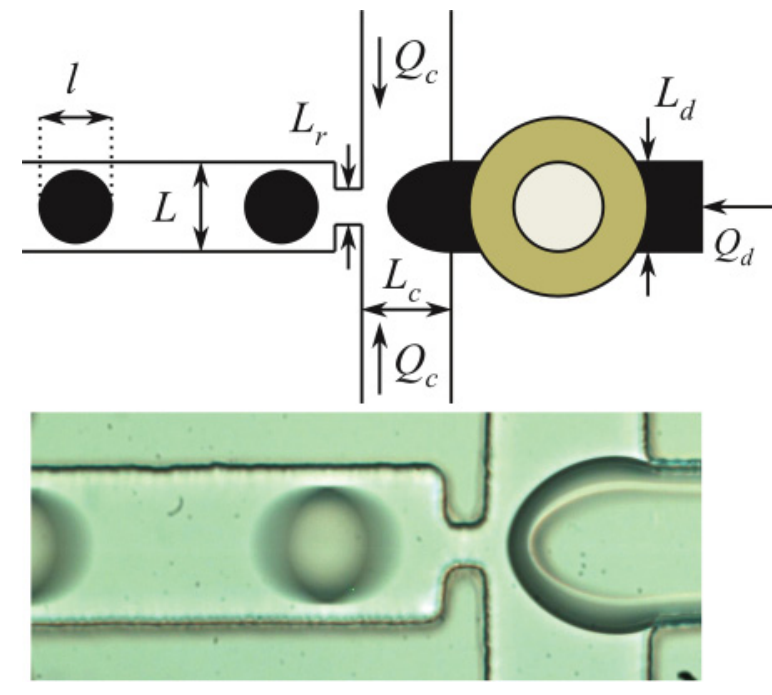

FIG. 1. (Color online) Device geometries of the flow-focusing junction fabricated with a piezoelectric disk (shape with two concentric circles). $L_{d}$ and $L_{c}$ represent the widths of the channel through which the dispersed phase and the continuous phase are flowing, respectively. $L_{r}$ represents the width of the orifice and $L$ represents the width of the outlet channel. $L_{d}=L_{c}=L \sim 150 \mu \mathrm{m}$ and $L_{r} \sim$ $40 \mu \mathrm{m} . Q_{d}$ and $Q_{c}$ represent the flow rates of the dispersed phase and the continuous phase, respectively. $l$ represents the length of the droplets formed.

He et al. [15] attempted to use an electric field in a flowfocusing microfluidic device to transform the flow front of the dispersed phase to a Taylor cone, which causes the droplet to break up due to the Rayleigh capillary instability. Submicron-sized droplets can be formed with this method, but the droplet size can only be controlled precisely at small flow-rate ratios. Nguyen et al. [16], Murshed et al. [17], and Tan et al. [18] developed thermally controlled droplet formation in flow-focusing and T-shaped geometries. This

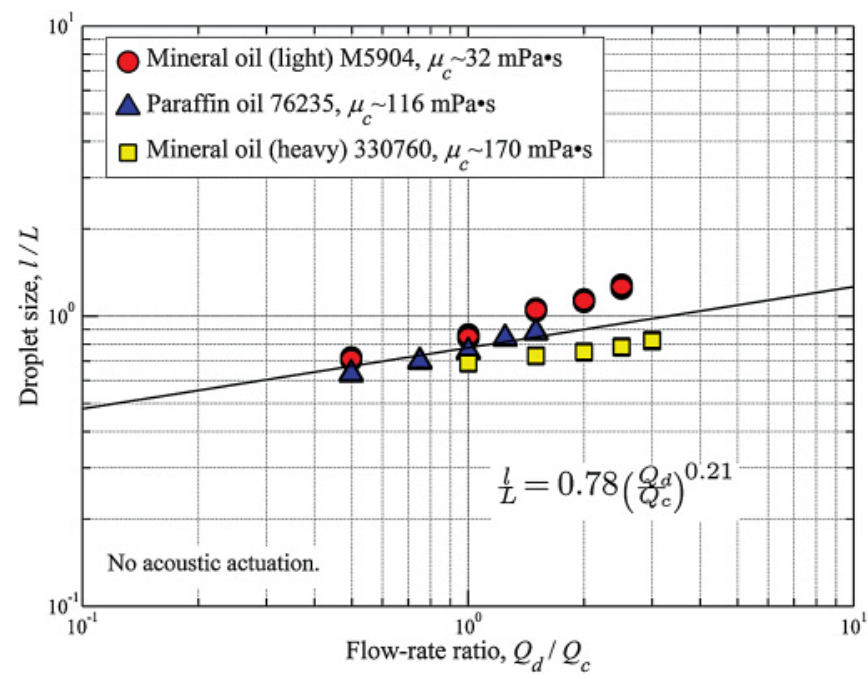

FIG. 2. (Color online) Characterization of the flow-focusing device showing the variation between the droplet size $l / L$ and the flow-rate ratio $\varphi$. Three types of oil with different dynamic viscosities as shown are investigated; the flow rate of the continuous phase is maintained at a constant value of $Q_{c}=100 \mu \mathrm{l} / \mathrm{h}$.
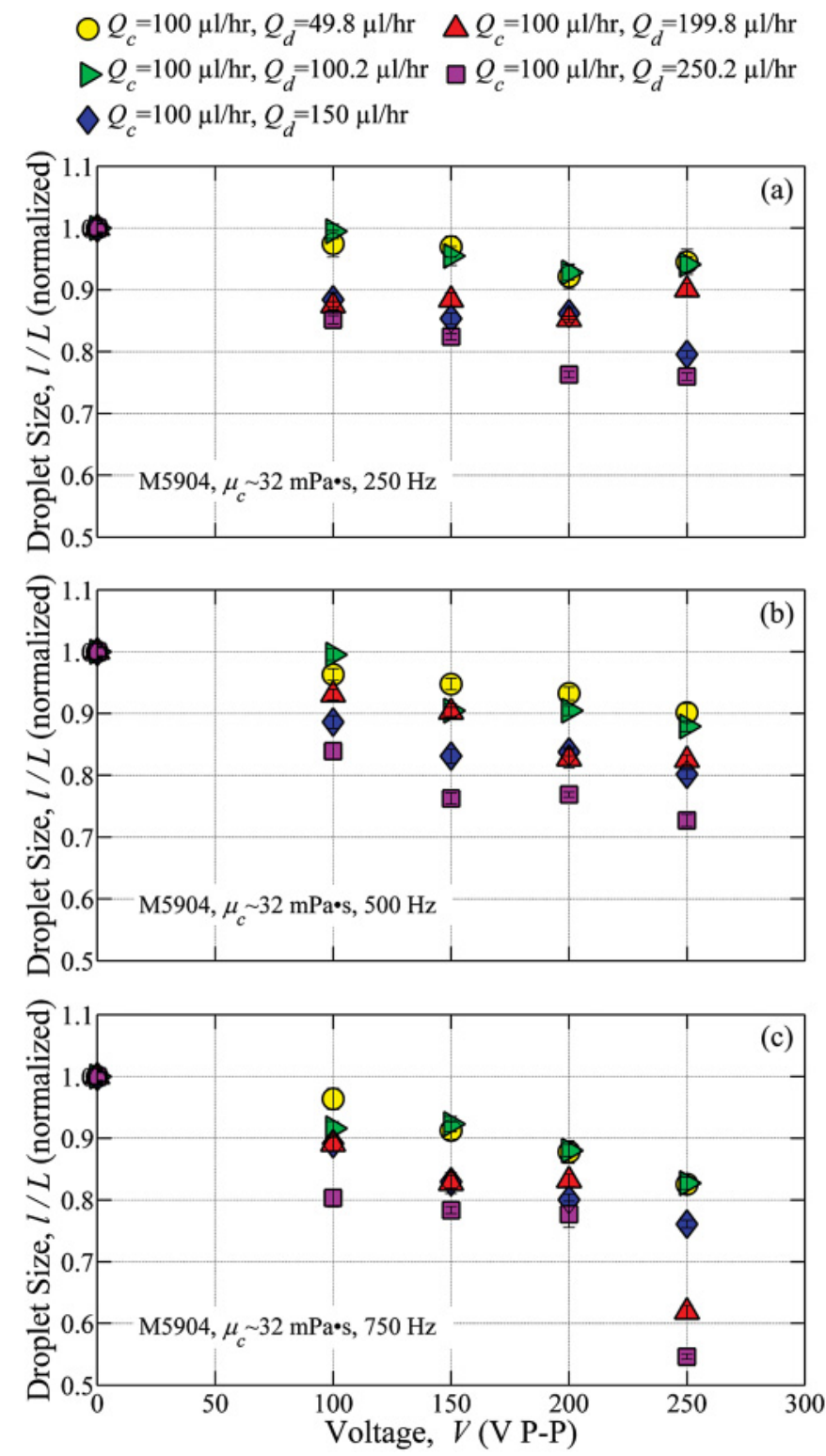

FIG. 3. (Color online) Variation of droplet size, $l / L$ (normalized by the initial value), with acoustic voltage applied at a fixed applied frequency for mineral oil (light) M5904 $\mu_{c} \sim 32 \mathrm{mPa}$.

method exploits the temperature-dependent nature of viscosity and interfacial tension to affect the breakup of droplets under preset temperatures. Droplet size increases with increasing temperature as both viscosity and interfacial tension decrease in value with an increase in temperature. Tan et al. [19] and Liu et al. [20] demonstrated the use of a magnetic field to affect the breakup of ferrofluid droplets. The size of the droplet formed depends on the magnetic-field strength and the location of the magnet, either upstream or downstream of the channel junction. However, this method is only applicable for fluids doped with ferromagnetic materials. Cheung and Qiu [21] demonstrated the preliminary results utilizing acoustic actuation to affect the breakup of droplets in a flow-focusing geometry. Droplet size decreases with increasing frequency of acoustic actuation for a specific applied voltage. The investigation was mainly focused on the squeezing regime 

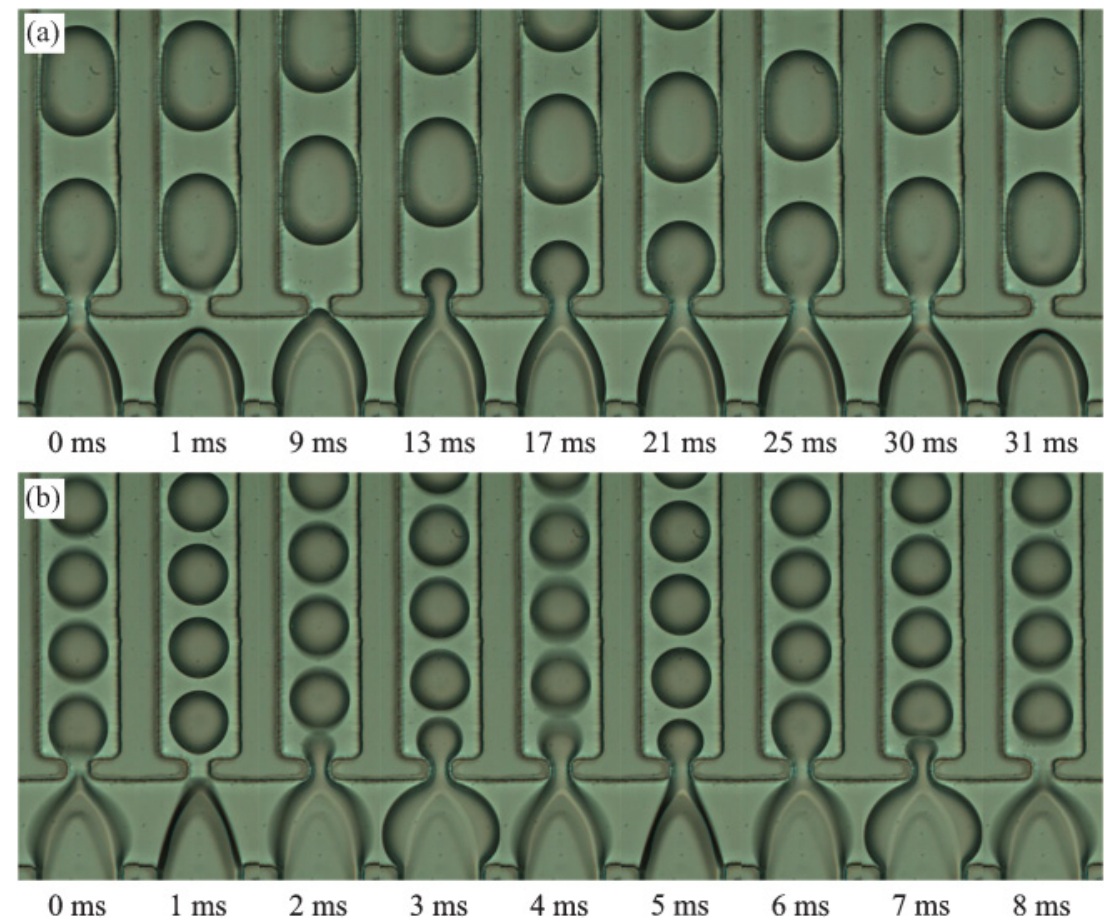

FIG. 4. (Color online) Phase diagrams of one droplet-formation process at the flowfocusing junction (a) without acoustic actuation and (b) with acoustic actuation at an applied voltage of $250 \mathrm{~V}$ peak-to-peak (P-P) sine wave at $750 \mathrm{~Hz}$. For both cases, the flow rates of mineral oil (light) M5904 and D.I. water are $Q_{c}=100$ $\mu \mathrm{l} / \mathrm{h}$ and $Q_{d}=250.2 \mu \mathrm{l} / \mathrm{h}$, respectively. Frames are taken at $1000 \mathrm{fps}$ with an exposure time of $250 \mu \mathrm{s}$. because the periodic oscillation from the piezoelectric actuator is effective in inducing an oscillating fluid-fluid interface, which affects the breakup of the liquid thread in this regime.

Acoustic actuation is a useful tool in the manipulation of fluid flow in microfluidic devices [22-25]. To study the flow fields in these microfluidic devices, $\mu$ PIV is adopted. However, this technique imposes extra difficulties in microscale situations, including intense lighting requirements with the use of small tracer particles and the scattering noise from the background. The situation may become more difficult when one encounters pulsating flows in which a periodic pulsating velocity superimposes on a constant velocity [26]. Tian and Qiu [27] improved the $\mu$ PIV technique to eliminate background scattering in microflow measurements. Phaseresolved PIV is a useful method in resolving the phaseaveraged, global-averaged, and periodic velocity components in the periodic flows [28,29].

In this study, the effects of the control parameters, flow-rate ratio, voltage and frequency of acoustic actuation, and continuous phases with different viscosities on droplet formation in a flow-focusing device were characterized. In addition, detailed $\mu$ PIV analysis was carried out to resolve the periodic velocity components of the acoustic actuation in a fluid medium.

\section{DROPLET SIZE CHARACTERIZATION UNDER ACOUSTIC ACTUATION}

\section{A. Device fabrication and experimental setup}

A flow-focusing device with geometric dimensions as shown in Fig. 1 was fabricated using the soft-lithography technique [30]. SU-8 (SU-8 2035, MicroChem Co.) was first spin-coated onto a silicon wafer and then exposed to ultraviolet (UV) light using a mask with the projected microchannel patterns. Polydimethylsiloxane (PDMS, SYLGARD ${ }^{\circledR} 184$, Dow Corning Co.) was used as the stamp resin to replicate the SU-8 channel patterns. The PDMS was first mixed with its hardener in a ratio of 10:1. The mixture was then degassed, poured into the SU-8 mold, and hardened in an oven. The hardened PDMS with microchannel patterns was punched to create inlet and outlet holes and then oxygen-plasma-bonded to a glass wafer to ensure the transparency of the device for observation under a microscope. A two-layer piezoelectric bending disk with a diameter of $12.7 \mathrm{~mm}$ and a thickness of $0.41 \mathrm{~mm}$ (T216-A4NO-273X, Piezo Systems, Inc.) was used. This piezoelectric disk was first cast into a PDMS substrate and then oxygen-plasma-bonded to the PDMS device with the microchannel patterns at a location upstream of the cross-junction. The depth of the channel $h$ was around $90 \mu \mathrm{m}$ and the thickness of the PDMS from the lower surface of the piezoelectric disk to the upper surface of the microchannel was around $1.7 \mathrm{~mm}$.

Visualization was performed with a microscope (Eclipse TE2000-U, Nikon Co.) attached with a high-speed camera (MotionXtra HG-100K, Redlake Co.). A sinusoidal signal

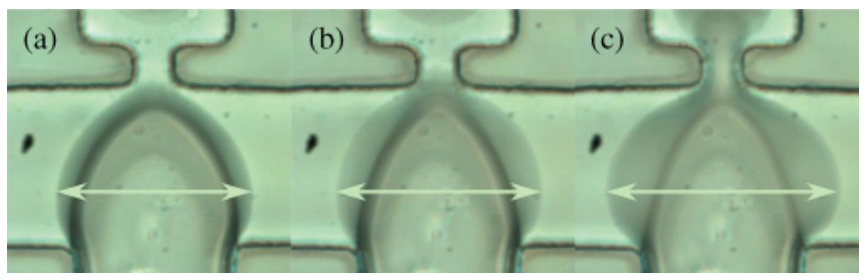

FIG. 5. (Color online) Vibrating interface between the two immiscible fluids, D.I. water, and mineral oil (light) M5904 $\mu_{c} \sim$ $32 \mathrm{mPa}$. The extent of vibration (as indicated by the double arrow) increases with an increase in applied voltage and frequency, $Q_{c}=100$ $\mu \mathrm{l} / \mathrm{h}$ and $Q_{d}=100.2 \mu \mathrm{l} / \mathrm{h}$ for all three cases. The conditions for the sine wave acoustic actuation are (a) $100 \mathrm{~V} \mathrm{P}-\mathrm{P}$ at $750 \mathrm{~Hz}$; (b) $250 \mathrm{~V}$ $\mathrm{P}-\mathrm{P}$ at $250 \mathrm{~Hz}$; and (c) $250 \mathrm{~V} \mathrm{P}-\mathrm{P}$ at $750 \mathrm{~Hz}$. 


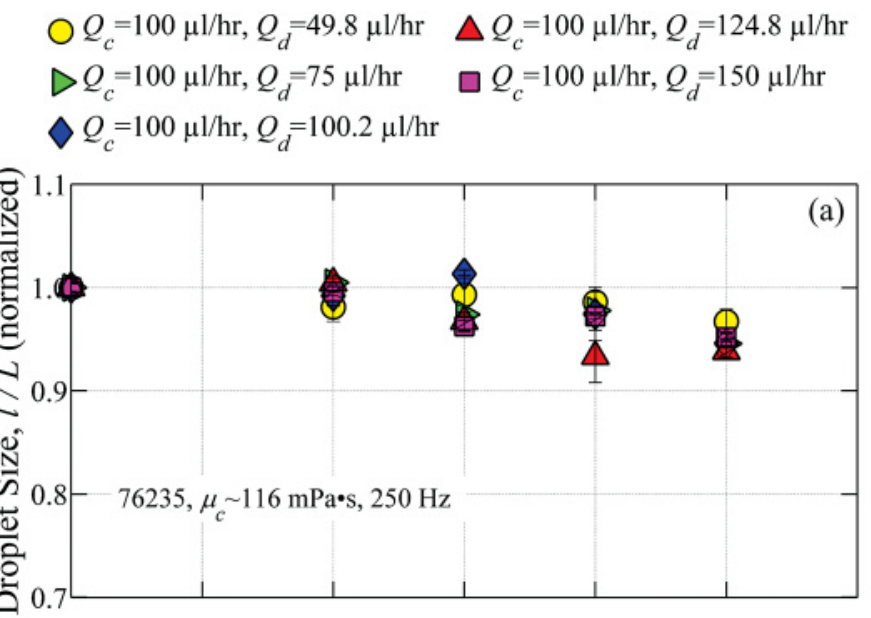

$$
\begin{aligned}
& \bigcirc Q_{c}=100 \mu \mathrm{l} / \mathrm{hr}, Q_{d}=100.2 \mu \mathrm{l} / \mathrm{hr} \quad \triangle Q_{c}=100 \mu \mathrm{l} / \mathrm{hr}, Q_{d}=250.2 \mu \mathrm{l} / \mathrm{hr} \\
& \triangleright Q_{c}=100 \mu \mathrm{l} / \mathrm{hr}, Q_{d}=150 \mu \mathrm{l} / \mathrm{hr} \quad \square Q_{c}=100 \mu \mathrm{l} / \mathrm{hr}, Q_{d}=300 \mu \mathrm{l} / \mathrm{hr} \\
& \diamond Q_{c}=100 \mu \mathrm{l} / \mathrm{hr}, Q_{d}=199.8 \mu \mathrm{l} / \mathrm{hr}
\end{aligned}
$$
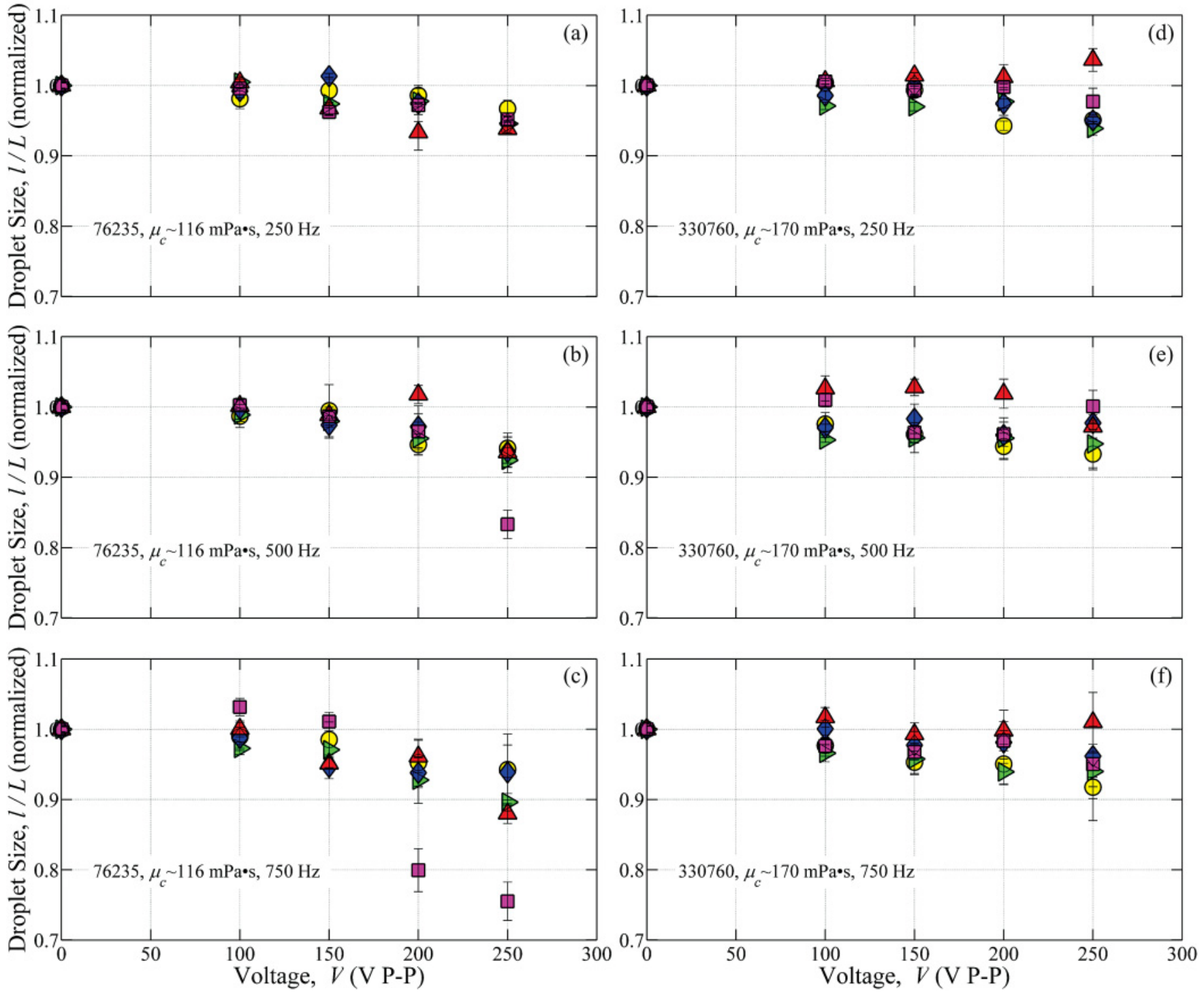

FIG. 6. (Color online) Variation of droplet size, $l / L$ (normalized by the initial value), with acoustic voltage applied at a fixed applied frequency for (a)-(c) paraffin oil $76235 \mu_{c} \sim 116 \mathrm{mPa} \cdot \mathrm{s}$ and (d)-(f) mineral oil (heavy) $330760 \mu_{c} \sim 170 \mathrm{mPa}$ s.

from a function generator (HP33120A, Hewlett-Packard Co.) was amplified by a high-speed bipolar amplifier (BA4825, NF Co.) and the signal was used to drive the piezoelectric disk. Three types of oils with different dynamic viscosities $\mu_{c}$ were used as the continuous phases. These oils were mineral oil (light) M5904 (SIGMA-ALDRICH ${ }^{\circledR}$ ) with $\mu_{c} \sim 32 \mathrm{mPa} \mathrm{s}$ $\left(20{ }^{\circ} \mathrm{C}\right)$, paraffin oil $76235\left(\right.$ FLUKA $\left.^{\circledR}\right)$ with $\mu_{c} \sim 116 \mathrm{mPa} \mathrm{s}$ $\left(20^{\circ} \mathrm{C}\right)$, and mineral oil (heavy) 330760 (SIGMAALDRICH $\left.^{\circledR}\right)$ with $\mu_{c} \sim 170 \mathrm{mPa} \mathrm{s}\left(20^{\circ} \mathrm{C}\right)$. Deionized (D.I.) water was used as the dispersed phase and the two liquids were driven by a twin syringe pump (Model 33, Harvard Apparatus Co.). The effects of the flow-rate ratio $\varphi$ of the two immiscible fluids, voltage $V$, and frequency $f$ of the acoustic actuation were applied, and the continuous phases with different viscosities were investigated. In all cases, the flow rate of the continuous phase $Q_{c}$ was maintained at a constant value of 100 $\mu \mathrm{l} / \mathrm{h}$ and, therefore, the capillary number $\mathrm{Ca}$ was constant. The length $l$ of each droplet produced was measured and normalized by the width of the outlet channel $l / L$ (Fig. 1). Characterization of the device was performed, and the results showing the variation between the droplet size $l / L$ and the flow-rate ratio $\varphi$ without acoustic actuation for the three continuous phases of different viscosities are shown in Fig. 2. Ten measurements were taken for each data point, and a conventional power-law relationship was calculated using the least-squares method.

\section{B. Results and discussion}

Characterization of the droplet size under acoustic actuation using the device and experimental setup described above was 


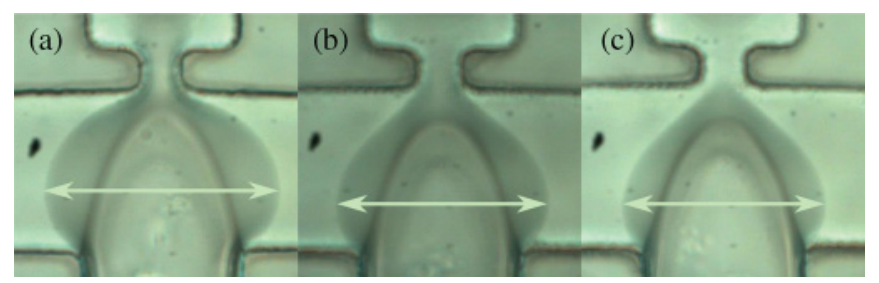

FIG. 7. (Color online) Vibrating interfaces between the two immiscible fluids (D.I. water and oils) under sine wave acoustic actuation of $250 \mathrm{~V} \mathrm{P}-\mathrm{P}$ at $750 \mathrm{~Hz} . Q_{c}=100 \mu \mathrm{l} / \mathrm{h}$ and $Q_{d}=100.2 \mu \mathrm{l} / \mathrm{h}$ for all three cases. The extent of vibration (as indicated by the double arrow) decreases with an increase in viscosity of the continuous phase $\mu_{c}$, (a) mineral oil (light) M5904 $\mu_{c} \sim 32 \mathrm{mPa}$, (b) paraffin oil 76235 $\mu_{c} \sim 116 \mathrm{mPa}$ s, and (c) mineral oil (heavy) $330760 \mu_{c} \sim 170 \mathrm{mPa}$ s. Pseudo-tip-streaming of the dispersed phase occurs under acoustic actuation and is more prominent for continuous phase with a higher viscosity.

performed, and the variation between the droplet size $l / L$, frequency $f$, and voltage $V$ for mineral oil (light) M5904 under acoustic actuation is shown in Fig. 3. Each data point is the average of ten measurements under the same experimental condition. As shown in Fig. 3, the droplet size formed under acoustic actuation decreases with the increase in both the voltage and frequency of the acoustic actuation applied. Such a phenomenon is due to the effect of the vibrating motion of the fluid-fluid interface on the whole droplet-formation process. The droplet-formation regime in the current investigation is the squeezing regime in which droplets are formed by the accompanying squeezing of the liquid thread near the orifice, and the eventual breakup of the liquid thread is through a series of equilibrium states. Phase diagrams showing one typical breakup process with and without acoustic actuation are shown in Fig. 4 for the case of $Q_{c}=100 \mu \mathrm{l} / \mathrm{h}$ and $Q_{d}=250.2 \mu \mathrm{l} / \mathrm{h}$. As shown in Fig. 4(a), each droplet-formation process starts with the advancement of the flow front of the dispersed phase up to the orifice (1-9 ms). The liquid thread then continues to fill up the orifice (9-30 ms) until the upstream pressure is high enough to trigger a continuous reduction in the width of the liquid thread near or within the orifice with a continuous increase in its axial curvature and the final breakup (30-31 ms) $[2,10,11]$. As a comparison, the phase diagram of the breakup of a droplet under the effect of acoustic actuation is shown in Fig. 4(b), in which a periodic expanding and contracting motion of the fluid-fluid interface and a periodic change in its curvature at the upstream of the orifice are indicated; the acoustic actuation is not only effective in reducing the time for the flow front of the dispersed phase to reach the orifice (1-2 ms), but it also reduces the time needed for the dispersed phase to fill up the orifice (2-6 ms) and for thinning of the liquid thread and finally its breakup (6-7 ms). There are two actions that facilitate the above changes. The first is the periodic pushing force provided by the piezoelectric actuator acting upon the dispersed phase, which accelerates the filling up of the orifice. The second is the periodic vibration motion and, therefore, the change in the curvature of the liquid-liquid interface upstream of the orifice, which causes a periodic pressure change acting upon the liquid thread and thus accelerating the trigger of its breakup. In addition, the continuous reduction in the droplet size with the increase in both the voltage and frequency of acoustic actuation applied can be explained by the observation that the extent of vibration motion of the fluid-fluid interface increases with both the increase in the voltage and the frequency applied under the same fluid flow condition as shown in Fig. 5.

Viscosity plays an important role in the volume of droplets formed in the squeezing regime in flow-focusing devices. Garstecki et al. [2,10] proposed a relationship between the volume of bubbles formed in a flow-focusing device $V_{b}$, the flow rate of the continuous phase $Q_{c}$, the viscosity of the continuous phase $\mu_{c}$, and the pressure of the dispersed phase $p_{d}$ as given by Eq. (4),

$$
V_{b} \propto \frac{p_{d}}{Q_{c} \mu_{c}},
$$

which shows that the volume of bubbles $V_{b}$ formed decreases with an increase in the viscosity of the continuous phase $\mu_{c}$. The above relationship is derived from three proportional relationships. The first one is between the volume of the bubbles $V_{b}$ formed and the product of the time for the liquid thread to pass through the orifice before breakup occurs $\tau$ and the flow rate of the dispersed phase $Q_{d}$ as given by Eq. (5),

$$
V_{b} \propto \tau Q_{d}
$$

where the time $\tau$ is inversely proportional to the flow rate of the continuous phase $Q_{c}$ as the evolution of the minimum width of the liquid thread during breakup is limited by the flow rate of the continuous phase $Q_{c}$. This is given by Eq. (6),

$$
\tau \propto \frac{1}{Q_{c}} .
$$

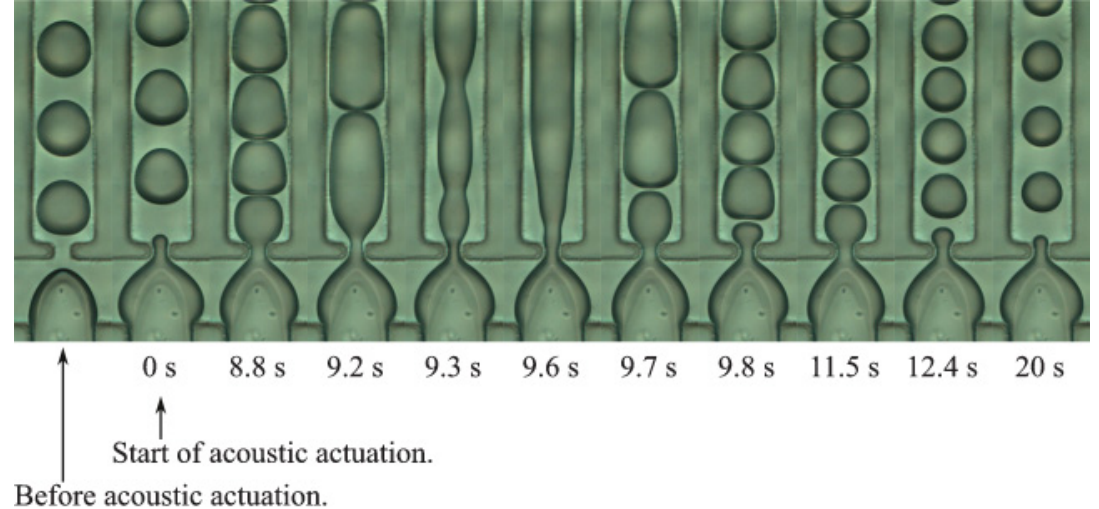

FIG. 8. (Color online) Phase diagram of flow regulation at the flow-focusing junction with acoustic actuation at an applied voltage of $250 \mathrm{~V}$ $\mathrm{P}-\mathrm{P}$ sine wave at $750 \mathrm{~Hz}$. The flow rates of paraffin oil 76235 and D.I. water are $Q_{c}=100 \mu \mathrm{l} / \mathrm{h}$ and $Q_{d}=150 \mu \mathrm{l} / \mathrm{h}$, respectively. Flow regulation occurs when the rate of droplet production is high enough to cause droplet coalescence downstream of the junction. 
During the bubble inflation process, the continuous phase is restricted to flow into the outlet channel and hence $Q_{d}$ is approximately the total flow rate in the outlet channel. Thus the third proportional relationship is the Hagen-Poiseuille relation, which is given by Eq. (7),

$$
Q_{d} \propto \frac{p_{d}}{\mu_{c}} .
$$

Equation (4) can be obtained by combining Eqs. (5)-(7). Nie et al. [31] showed that the viscosity of the dispersed phase $\mu_{d}$ affects the size of the droplet formed, and the dependence of the volume of droplets produced on the flow-rate ratio $\varphi$ between the two immiscible fluids decreases as the viscosity of the dispersed phase increases.

In the current investigation, the effect of viscosity of the continuous phase $\mu_{c}$ on droplet formation under acoustic actuation is studied with three types of oil, and the results are shown in Figs. 3 and 6. According to the figures, acoustic actuation is more effective in reducing the size of droplets formed for a continuous phase with a lower viscosity as the relative change in droplet size is larger for mineral oil (light), M5904 $\mu_{c} \sim 32 \mathrm{mPa}$ s, than for paraffin oil, $76235 \mu_{c} \sim$ $116 \mathrm{mPa}$. However, there is almost no obvious relative change in droplet size for mineral oil (heavy), $330760 \mu_{c} \sim 170 \mathrm{mPa}$. As discussed above, the effectiveness of the acoustic actuation on the breakup of droplets depends mainly on the extent of vibration motion of the fluid-fluid interface at the cross- junction of the device. Figure 7 shows three images indicating a decrease in the extent of vibration motion as the viscosity of the continuous phase $\mu_{c}$ increases. The increase in the shear stress from the continuous phase with a higher viscosity limits the extent of the vibration motion of the interface, and thus acoustic actuation is more effective in affecting the breakup of the dispersed phase when the continuous phase has a low viscosity. In addition, the high shear stress from the viscous fluid induces a pseudo-tip-streaming phenomenon as shown in Fig. 7(c). Furthermore, the acoustic actuation applied causes an increase in the rate of droplet formation, and we observed a few cases in which the rate of the droplet breakup is faster than the velocity of the droplet flow downstream of the orifice. Droplet coalescence occurs and flow regulation starts until a new stable breakup rate is achieved (Fig. 8).

\section{PHASE-AVERAGED $\mu$ PIV ANALYSIS}

The periodic nature of the oscillating flow field can be studied using phase-averaged $\mu \mathrm{PIV}$, and this technique is effective in extracting the organized motion from the total signal. The fluctuating fluid flow velocities $\vec{u}(\vec{x}, t)$ at a particular location $\vec{x}$ and time $t$ can be decomposed into three components [32] as given by Eq. (8),

$$
\vec{u}(\vec{x}, t)=\overline{\vec{u}}(\vec{x})+\tilde{\vec{u}}(\vec{x}, t)+\vec{u}^{\prime}(\vec{x}, t),
$$
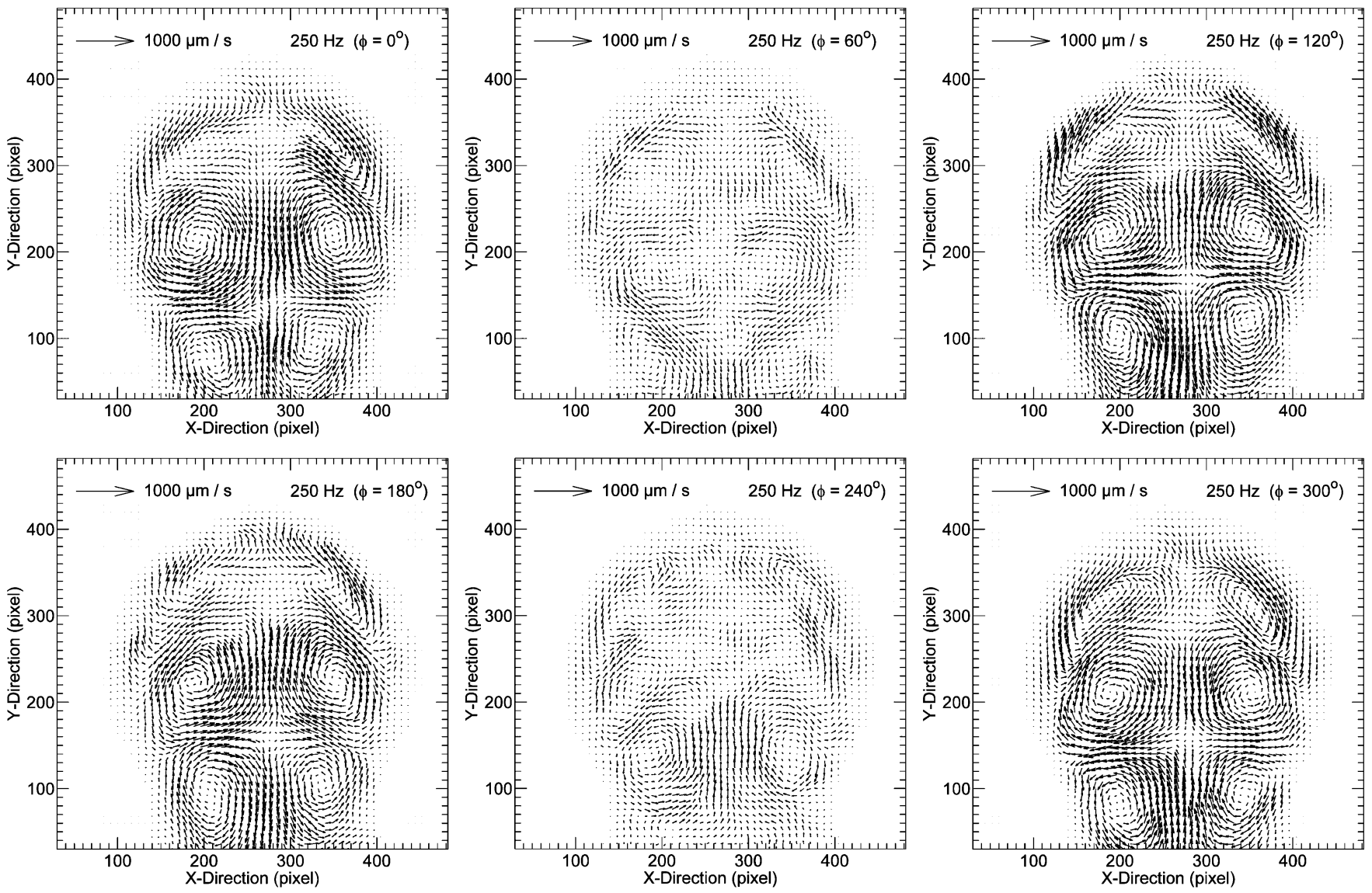

FIG. 9. Periodic velocity components in one actuation cycle for acoustic sine wave actuation of $300 \mathrm{~V} \mathrm{P}-\mathrm{P}$ at $250 \mathrm{~Hz}$ with phases $\phi=0^{\circ}$, $60^{\circ}, 120^{\circ}, 180^{\circ}, 240^{\circ}$, and $300^{\circ} . Q_{c}=100 \mu \mathrm{l} / \mathrm{h}$ and $Q_{d}=30 \mu \mathrm{l} / \mathrm{h}$. 

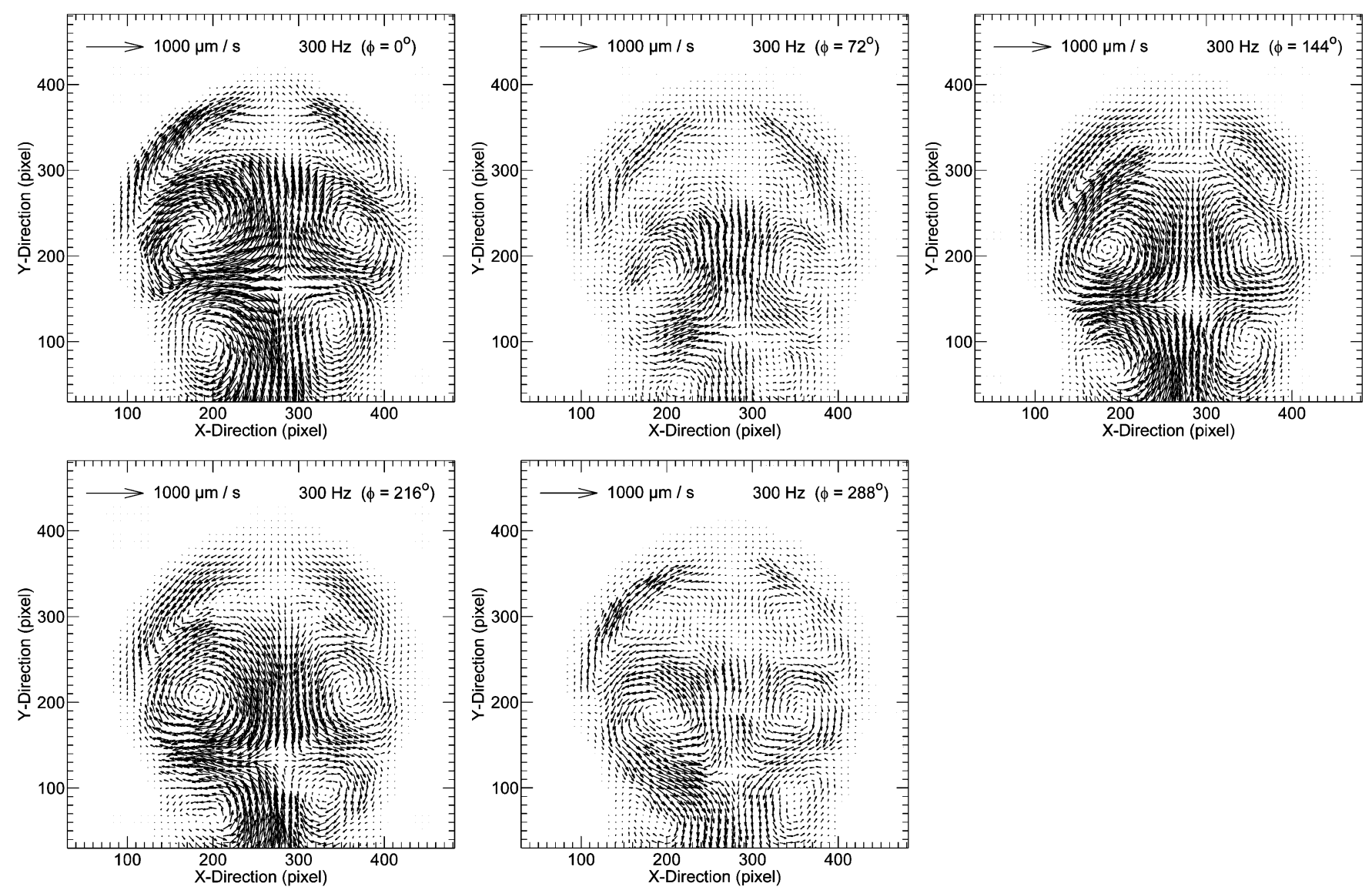

FIG. 10. Periodic velocity components in one actuation cycle for acoustic sine wave actuation of $300 \mathrm{~V}$ P-P at $300 \mathrm{~Hz}$ with phases $\phi=0^{\circ}$, $72^{\circ}, 144^{\circ}, 216^{\circ}$, and $288^{\circ} . Q_{c}=100 \mu \mathrm{l} / \mathrm{h}$ and $Q_{d}=30 \mu \mathrm{l} / \mathrm{h}$.

where $\overline{\vec{u}}(\vec{x})$ is the global-averaged (time-averaged) component, $\tilde{\vec{u}}(\vec{x}, t)$ is the periodic component, and $\vec{u}(\vec{x}, t)$ is the random component. In order to obtain all the components, the relations as described in Eqs. (9) and (10) are used,

$$
\begin{gathered}
\tilde{\vec{u}}(\vec{x}, t)=\langle\vec{u}(\vec{x}, t)\rangle-\overline{\vec{u}}(\vec{x}), \\
\vec{u}^{\prime}(\vec{x}, t)=\vec{u}(\vec{x}, t)-\langle\vec{u}(\vec{x}, t)\rangle,
\end{gathered}
$$

where $\langle\vec{u}(\vec{x}, t)\rangle$ is the phase-averaged component and is given by Eq. (11):

$$
\langle\vec{u}(\vec{x}, t)\rangle=\lim _{N \rightarrow \infty} \frac{1}{N} \sum_{j=1}^{N} \vec{u}[\vec{x}, t+(j-1) T],
$$

where $N$ is the number of cycles and $T$ is the period of the oscillation motion or the period of the acoustic actuation applied as in the current investigation. An estimate of the phase-averaged component for a particular phase $\langle\vec{u}(\vec{x}, t)\rangle_{p \text { (estimate) }}$ can be obtained by Eq. (12) based on the average of a finite number of $N_{p}$ cycles at phase $p$,

$$
\langle\vec{u}(\vec{x}, t)\rangle_{p \text { (estimate) }}=\frac{1}{N_{p}} \sum_{j=1}^{N_{p}} \vec{u}[\vec{x}, t+(j-1) T] .
$$

The estimate of the global-averaged component is the average of all the phase-averaged components and is given by Eq. (13),

$$
\overline{\vec{u}}(\vec{x})_{(\text {estimate })}=\frac{1}{P} \sum_{p=1}^{P}\langle\vec{u}(\vec{x}, t)\rangle_{p(\text { estimate })},
$$

where $P$ is the total number of phases.

\section{A. Device fabrication and experimental setup}

A flow-focusing device with the same geometrical arrangements as the one shown in Fig. 1 and with a channel depth $h$ of around $100 \mu \mathrm{m}$ was fabricated. A simple piezoelectric disk, which is usually used in a buzzer system, was first cast into a PDMS substrate, which was then bonded to the flow-focusing device at a location upstream of the flow-focusing junction. The thickness of the PDMS from the lower surface of the piezoelectric disk to the upper surface of the microfluidic channel is around $1.1 \mathrm{~mm}$. Mineral oil (light) M5904 $\mu_{c} \sim$ $32 \mathrm{mPa}$ s was used as the continuous phase and D.I. water was used as the dispersed phase. The flow rate of the continuous phase was $Q_{c}=100 \mu \mathrm{l} / \mathrm{h}$ and that of the dispersed phase was $Q_{d}=30 \mu \mathrm{l} / \mathrm{h}$.

$\mu$ PIV measurements were conducted with an intensified high-speed camera (Ultracam3, Phantom ${ }^{\circledR}$, OPELCO Co.) that captured fluorescent particle images at a preset framing rate. The intensified camera was attached to the microscope (Eclipse TE2000-U, Nikon Co.) on which the microfluidic device was placed for observation. In addition, a $20 \times$ with 
a $1.5 \times$ intermediate magnification objective was used (Plan Fluor., ELWD, $20 \times / 0.45$, Nikon Co.). The dispersed phase was doped with $1 \mu \mathrm{m}$ red fluorescent particles (R0100B, Thermo Scientific Co.) in a volume concentration of $0.25 \%$, which resulted in around $6 \times 10^{8}$ particles per milliliter. Light illumination from a super-high-pressure mercury lamp (C-SHG1 for HG-100W, Nikon Co.) passed through a filter cube with an excitation filter (510-560 nm), a dichromatic mirror $(575 \mathrm{~nm})$, and a barrier filter $(590 \mathrm{~nm})$. Finally, the light of the red fluorescent particles (612 nm maximum) was captured by the intensified camera. Cross-correlation calculation based on the captured images was carried out using commercial PIV software (DaVis 6.2, LaVision Co.). The multipass second-order correlation algorithm with a decreasing interrogation window size and $50 \%$ window overlap was adopted. The calculation was carried out with two iterations of the initial interrogation window size of $32 \times 32$ pixel $^{2}$ and finally one iteration of the interrogation window size of $16 \times 16$ pixel $^{2}$, resulting in around three fluorescent particles per interrogation window volume $\left(18 \times 18 \times 14 \mu \mathrm{m}^{3}\right)$. In the current investigation, an acoustic sine wave electrical signal used to drive the piezoelectric disk was produced by a function generator (HP33120A, Hewlett-Packard Co.) and was then amplified by a high speed bipolar amplifier (BA4825, NF Co.) up to a voltage of $300 \mathrm{~V} \mathrm{P-P}$ at frequencies of 250 and $300 \mathrm{~Hz}$. A speed of $3000 \mathrm{fps}$ with an exposure time of $35 \mu \mathrm{s}$ was chosen as the framing rate of the intensified camera, which resulted in 12 and 10 phases within one actuation cycle for the 250 and $300 \mathrm{~Hz}$ cases, respectively. In the calculation of the phase-averaged components, 15 image pairs were used for the correlation field averaging of each phase, and a total of 180 and 150 image pairs were used for the calculation of global-averaged components for 250 and $300 \mathrm{~Hz}$, respectively.

\section{B. Results and discussion}

Details on the phase-averaged microstreaming vector fields can be found in the results of Cheung and Qiu [21]. Such a microstreaming flow pattern is induced by the perpendicular vibrating motion at the boundary upstream of the

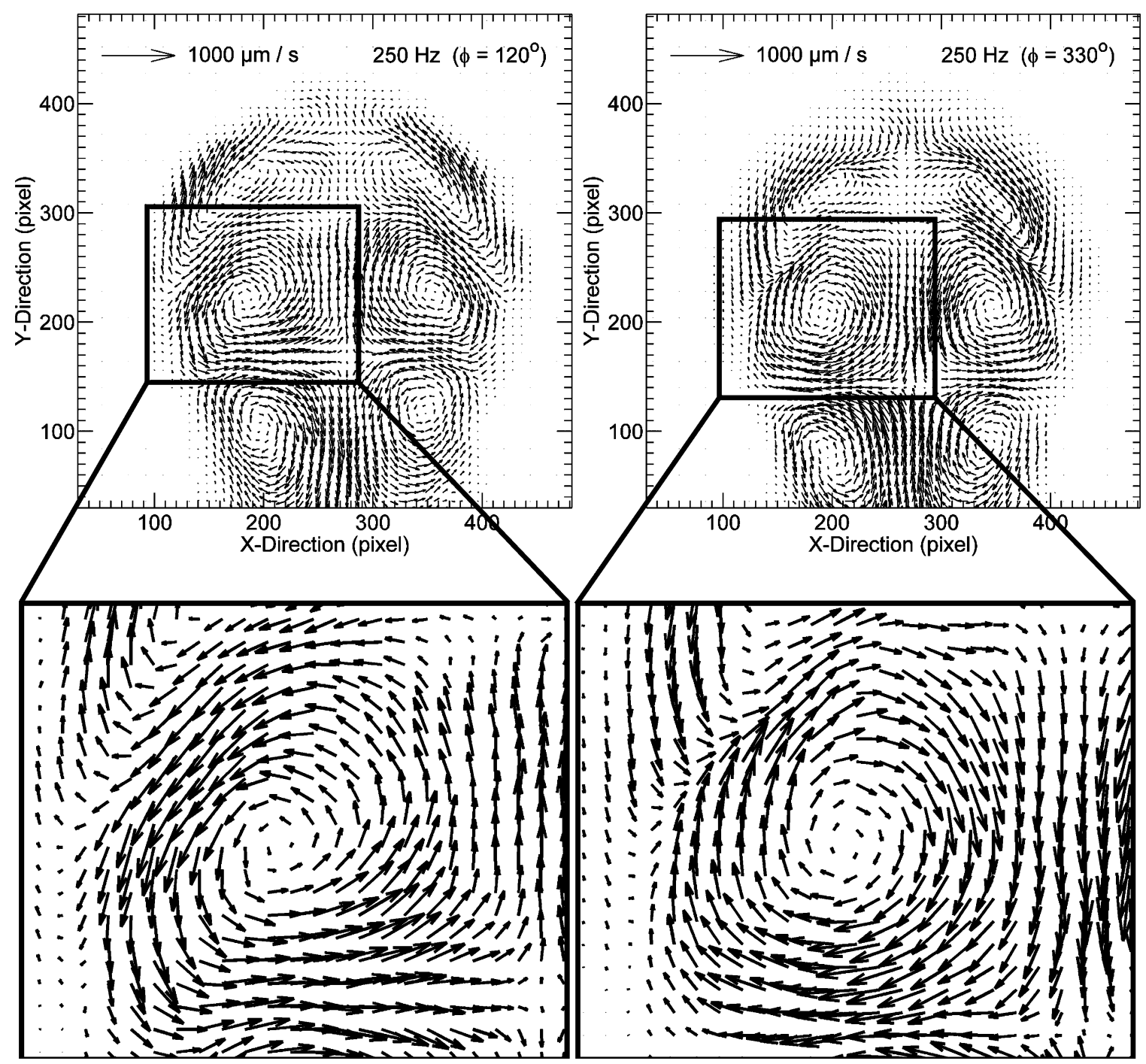

FIG. 11. Counter-rotating vortices at two different phases $\left(\phi=120^{\circ}\right.$ and $\left.330^{\circ}\right)$ for the $300 \mathrm{~V}$ P-P at $250 \mathrm{~Hz}$ acoustic actuation in one actuation cycle. $Q_{c}=100 \mu \mathrm{l} / \mathrm{h}$ and $Q_{d}=30 \mu \mathrm{l} / \mathrm{h}$. 
cross-junction of the PDMS device. The attenuation of the acoustic wave leads to the creation of Reynolds stress, which is the force driving the acoustic streaming flow [33]. The scale of this boundary-induced streaming flow becomes more prominent in microfluidic devices as the streaming force generated by the vibrating boundary is independent of the channel size [34]. The stratified flow structure of the phaseaveraged components shows the interaction of the shearing flow motion at the interface between the continuous phase and the dispersed phase as well as the interaction between the shearing flow and the microstreaming flow within the dispersed phase. The periodic components for $6\left(\phi=0^{\circ}, 60^{\circ}, 120^{\circ}\right.$, $180^{\circ}, 240^{\circ}$, and $300^{\circ}$ ) out of 12 phases for the $250 \mathrm{~Hz}$ case and $5\left(\phi=0^{\circ}, 72^{\circ}, 144^{\circ}, 216^{\circ}\right.$, and $\left.288^{\circ}\right)$ out of 10 phases for the $300 \mathrm{~Hz}$ case calculated based on Eqs. (9), (12), and (13) are shown in Figs. 9 and 10. These components exhibit a stratified flow structure with the upper portion being a shearing layer between the two immiscible fluids and the lower portion

(a)

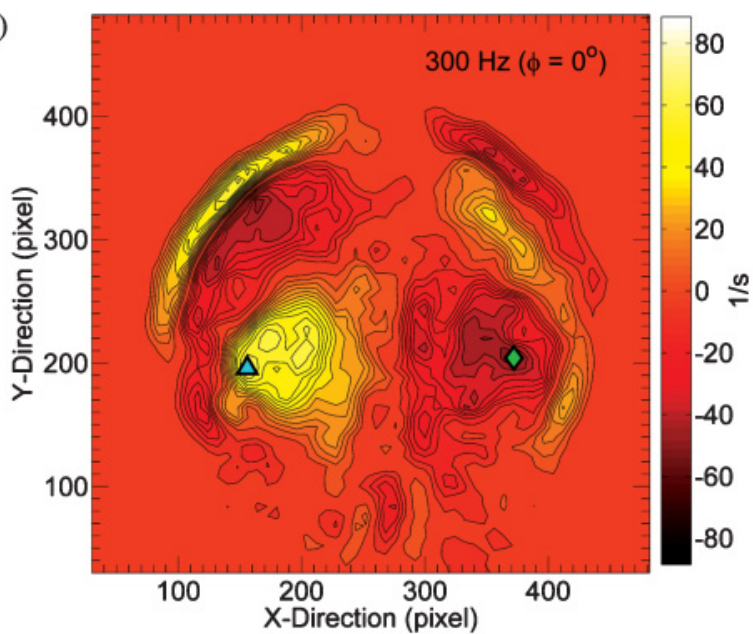

(b)
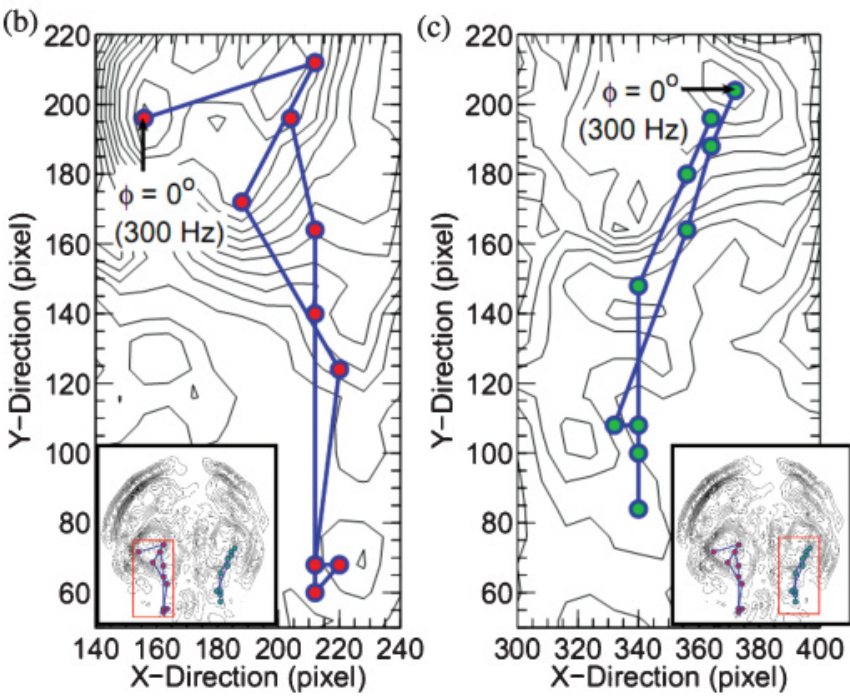

FIG. 12. (Color online) (a) Centers of maximum positive (cyan triangle) and negative (green diamond) vorticities of the phaseaveraged components for sine wave acoustic actuation of $300 \mathrm{~V}$ P-P at $300 \mathrm{~Hz}$. (b) Oscillation path for the points of maximum positive vorticities and (c) that of maximum negative vorticities for the 10 phases in one actuation cycle. $Q_{c}=100 \mu \mathrm{l} / \mathrm{h}$ and $Q_{d}=30 \mu \mathrm{l} / \mathrm{h}$. being a four-vortex structure with a flow separation point at the center. The organized periodic components are undergoing a periodic change in magnitude (as indicated by the length of the velocity vectors) and flow vortex direction in both the interface shearing flow and the central vortical flow. Figure 11 shows two enlarged counter-rotating vortices at two different phases in the same actuation cycle for the $250 \mathrm{~Hz}$ case. The periodicity of the periodic velocity components is the same as that of the vibrating interface, as shown in Figs. 4(b), 5, and 7. In addition, the oscillation motion at the point of maximum vorticity of the phase-averaged components as calculated by Eq. (12) can be traced by locating and plotting the points of maximum positive and negative vorticities $\vec{\omega}_{p}$ at phase $p$,

$$
\vec{\omega}_{p}=(\vec{\nabla} \times \vec{u})_{p},
$$

where $\vec{\omega}_{p}$ is calculated using the central difference scheme. Figure 12 shows the points of maximum positive and negative vorticities of the 10 phases in one actuation cycle for the case of $300 \mathrm{~V}$ P-P at $300 \mathrm{~Hz}$ acoustic actuation and the corresponding oscillation paths. The dominant motion direction of the maximum vorticity is in the $y$ direction, but the dominant expanding and contracting direction of the fluid-fluid interface [Figs. 4(b), 5, and 7] is in the $x$ direction.

\section{CONCLUSIONS}

Droplet breakup in flow-focusing devices can be divided into three main regimes: squeezing, dripping, and jetting. The different breakup mechanisms in these three regimes affect the choice of the local control methods used to manipulate the breakup of droplets in addition to the control by the flow-rate ratio between the two immiscible fluids. In this paper, the acoustic actuation affected breakup in the squeezing regime is studied. This regime is characterized by three stages of breakup: the first stage is the advancement of the flow front of the dispersed phase into the orifice; the second stage is the droplet inflation downstream of the orifice and the pressure buildup upstream and within the orifice; the third stage is the thinning and finally the breakup of the liquid thread. Acoustic actuation from the piezoelectric actuator induces oscillations at the fluid-fluid interface at the cross-junction of the device, and, therefore, it induces a periodic change in the curvature of the interface and internal vortex. This oscillation motion affects the total duration of droplet formation with significant time reduction in the first two stages of the whole breakup process, which results in a reduction in the droplet size. The pushing force from the piezoelectric disk facilitates the flow front of the dispersed phase to enter the orifice, and the vibrating motion of the fluid-fluid interface facilitates the onset of thinning of the liquid thread.

The effects of viscosity of the continuous phase play an important role in droplet formation in a flow-focusing device under acoustic actuation. Droplet formation under acoustic actuation is mainly affected by the extent of the vibration motion at the fluid-fluid interface. Using a higher viscosity liquid of continuous phase will induce higher shear stress to the dispersed phase, which results in a restrained vibration motion at the fluid-fluid interface. Hence, acoustic actuation is more effective in affecting the breakup of droplets for a continuous phase with lower viscosity. Detailed analysis of 
the relationship between the ratio of viscosity of the two immiscible fluids and the extent of the vibration motion caused by the acoustic actuation is helpful to understand the mechanism of the effects of viscosity on the breakup of the liquid thread.

The analysis of the oscillating flow fields under acoustic actuation is achieved using the method of phase-averaged $\mu \mathrm{PIV}$. Periodic flow components at different phases are resolved from the total velocity flow field based on the phase-averaged flow components and the global-averaged flow component from measured flow data. A stratified flow structure with a shearing layer near the interface between the two immiscible fluids and a four-vortex structure with a flow separation point at the center near the side poles of the cross-junction are obtained for the periodic components. These organized periodic components are undergoing a periodic change in both the flow vortex direction and the flow magnitude at the same frequency as that of the vibration of the fluid-fluid interface. The oscillation paths for the points of maximum positive and negative vorticities of the phase-averaged components are traced and the directions of oscillation are mainly along the $y$ direction; in contrast, the vibration of the fluid-fluid interface is mainly along the $x$ direction. The dynamics of internal flow vorticity and the switching vortex direction are the additional forces causing the periodic breakup of droplets.

\section{ACKNOWLEDGMENTS}

The authors acknowledge support for the current research from the Research Grants Council (RGC) of the Government of Hong Kong Special Administrative Region (HKSAR) under Projects No. 618508 and No. 618907.
[1] S. L. Anna, N. Bontoux, and H. A. Stone, Appl. Phys. Lett. 82, 364 (2003).

[2] P. Garstecki, I. Gitlin, W. DiLuzio, and G. M. Whitesides, Appl. Phys. Lett. 85, 2649 (2004).

[3] Q. Xu and M. Nakajima, Appl. Phys. Lett. 85, 3726 (2004).

[4] T. Ward, M. Faivre, M. Abkarian, and H. A. Stone, Electrophoresis 26, 3716 (2005).

[5] T. Cubaud, M. Tatineni, X. Zhong, and C.-M. Ho, Phys. Rev. E 72, 037302 (2005).

[6] P. Garstecki, M. J. Fuerstman, H. A. Stone, and G. M. Whitesides, Lab Chip 6, 437 (2006).

[7] A. M. Gañán-Calvo, Phys. Rev. E 69, 027301 (2004).

[8] J. H. Xu, S. W. Li, J. Tan, and G. S. Luo, Microfluid. Nanofluid. 5, 711 (2008).

[9] G. F. Christopher, N. N. Noharuddin, J. A. Taylor, and S. L. Anna, Phys. Rev. E 78, 036317 (2008).

[10] P. Garstecki, H. A. Stone, and G. M. Whitesides, Phys. Rev. Lett. 94, 164501 (2005).

[11] S. L. Anna and H. C. Mayer, Phys. Fluids 18, 121512 (2006).

[12] P. Garstecki, M. J. Fuerstman, and G. M. Whitesides, Phys. Rev. Lett. 94, 234502 (2005).

[13] L. Rayleigh, Proc. R. Soc. London 29, 71 (1879).

[14] H. Kim, D. Luo, D. Link, D. A. Weitz, M. Marquez, and Z. Cheng, Appl. Phys. Lett. 91, 133106 (2007).

[15] P. He, H. Kim, D. Luo, M. Marquez, and Z. Cheng, Appl. Phys. Lett. 96, 174103 (2010).

[16] N.-T. Nguyen, T.-H. Ting, Y.-F. Yap, T.-N. Wong, J. C.-K. Chai, W.-L. Ong, J. Zhou, S.-H. Tan, and L. Yobas, Appl. Phys. Lett. 91, 084102 (2007).

[17] S. M. S. Murshed, S. H. Tan, N. T. Nguyen, T. N. Wong, and L. Yobas, Microfluid. Nanofluid. 6, 253 (2009).
[18] S.-H. Tan, S. M. S. Murshed, N.-T. Nguyen, T. N. Wong, and L. Yobas, J. Phys. D 41, 165501 (2008).

[19] S.-H. Tan, N.-T. Nguyen, L. Yobas, and T. G. Kang, J. Micromech. Microeng. 20, 045004 (2010).

[20] J. Liu, S.-H. Tan, Y. F. Yap, M. Y. Ng, and N.-T. Nguyen, Microfluid. Nanofluid. 11, 177 (2011).

[21] Y. N. Cheung and H. H. Qiu, Appl. Phys. Lett. 97, 133111 (2010).

[22] X. Qu and H. H. Qiu, Heat Transf. Eng. 32, 636 (2011).

[23] J. Xu and D. Attinger, Phys. Fluids 19, 108107 (2007).

[24] J. Xu and D. Attinger, J. Micromech. Microeng. 17, 609 (2007).

[25] X. Qu and H. H. Qiu, J. Micromech. Microeng. 20, 095012 (2010).

[26] M. Nabavi and K. Siddiqui, Meas. Sci. Technol. 21, 042002 (2010).

[27] J. D. Tian and H. H. Qiu, Appl. Opt. 41, 6849 (2001).

[28] J. Sung and J. Y. Yoo, Meas. Sci. Technol. 12, 655 (2001).

[29] Y. S. Tsai, Y. M. Chang, Y. J. Chang, and Y. M. Chen, J. Fluid. Struct. 23, 191 (2007).

[30] J. C. McDonald, D. C. Duffy, J. R. Anderson, D. T. Chiu, H. Wu, O. J. A. Schueller, and G. M. Whitesides, Electrophoresis 21, 27 (2000).

[31] Z. Nie, M. Seo, S. Xu, P. C. Lewis, M. Mok, E. Kumacheva, G. M. Whitesides, P. Garstecki, and H. A. Stone, Microfluid. Nanofluid. 5, 585 (2008).

[32] W. C. Reynolds and A. K. M. F. Hussain, J. Fluid Mech. 54, 263 (1972).

[33] J. Lighthill, J. Sound Vib. 61, 391 (1978).

[34] K. D. Frampton, S. E. Martin, and K. Minor, Appl. Acoust. 64, 681 (2003). 